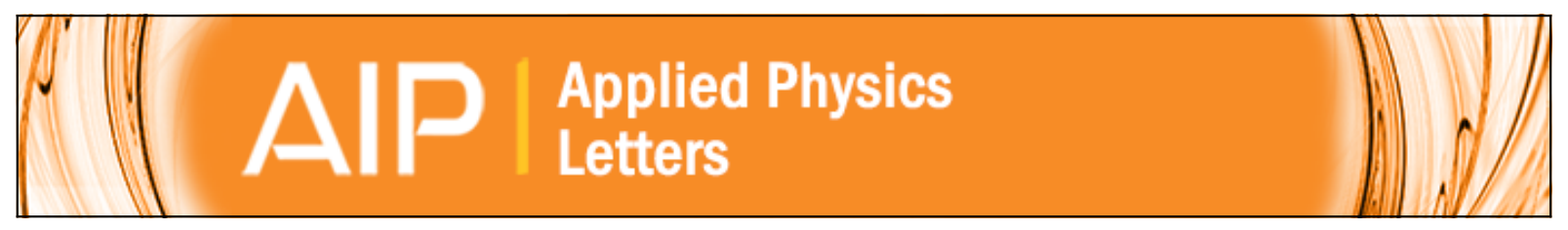

Charged defects in highly emissive organic wide-band-gap semiconductors

E. J. W. List, C. H. Kim, J. Shinar, A. Pogantsch, G. Leising, and W. Graupner

Citation: Applied Physics Letters 76, 2083 (2000); doi: 10.1063/1.126262

View online: http://dx.doi.org/10.1063/1.126262

View Table of Contents: http://scitation.aip.org/content/aip/journal/apl/76/15?ver=pdfcov

Published by the AIP Publishing

Over 700 papers \&

presentations on

multiphysics simulation new now

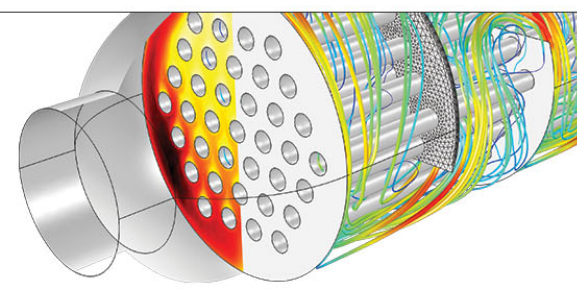




\title{
Charged defects in highly emissive organic wide-band-gap semiconductors
}

\author{
E. J. W. List ${ }^{\text {a) }}$ \\ Institut für Festkörperphysik, Technische Universität Graz, A-8010 Graz, Austria \\ and Ames Laboratory-USDOE, Iowa State University, Ames, Iowa 50011 \\ C. H. Kim and J. Shinar \\ Ames Laboratory-USDOE and Department of Physics and Astronomy, Iowa State University, \\ Ames, Iowa 50011
}

\author{
A. Pogantsch and G. Leising \\ Institut für Festkörperphysik, Technische Universität Graz, A-8010 Graz, Austria
}

W. Graupner

Department of Physics, Virginia Tech, Blacksburg, Virginia 24060

(Received 23 September 1999; accepted for publication 14 February 2000)

\begin{abstract}
A combined photoluminescence (PL) -detected magnetic-resonance (PLDMR) and thermally stimulated current (TSC) study of defects in wide-band-gap para-phenylene-type semiconductors is described. As TSC probes the density of mobile charge carriers after detrapping and PLDMR reveals the influence of trapped charges on the PL, their combination yields the concentration of traps, their energetic position, and their contribution to PL quenching. The reported trap densities, which are $2 \times 10^{16}$ for the polymer and $1 \times 10^{14} \mathrm{~cm}^{-3}$, for the oligomer, are the lowest reported for para-phenylene-type materials. (C) 2000 American Institute of Physics. [S0003-6951(00)03615-9]
\end{abstract}

Wide-band-gap para-phenylene-type semiconductors are both of fundamental interest for their rich photophysics ${ }^{1}$ and of commercial importance for applications such as organic light-emitting devices (OLEDs) (Ref. 2) and as active materials in lasers, ${ }^{3}$ which may also find applications in electrically pumped laser diodes (LDs). Electronic defects such as traps strongly influence, or even dominate, both the optical and charge transport properties of organic ${ }^{4}$ and inorganic devices. ${ }^{5}$ Charges trapped at these defects are missing as mobile carriers, ${ }^{6}$ change the space charge of the defect-rich region, can quench the emissive singlet excitons (SEs), ${ }^{7}$ and can reabsorb the emission. The latter two processes can introduce sufficient optical loss in a LD to prevent laser operation. ${ }^{7,8}$

In conjugated materials one has to distinguish between intrinsic electronic defects, such as polymer ends, and extrinsic defects, such as carbonyl groups. However, extrinsic defects in organic materials are as inevitable as impurities in inorganic materials, which are, e.g., responsible for the phenomenon of residual resistivity, ${ }^{5}$ which is a very important parameter in the characterization of pure metals. Therefore, studying device structures which are prepared under identical conditions as OLEDs with thermally stimulated current (TSC), elucidates intrinsic and extrinsic properties relevant for efficient device operation. In addition, introducing extrinsic defects deliberately, such as carbonyl groups by photooxidation, provides further insight into their role of electronic defects. This letter describes a combined charge transport and optical study of electronic defects in paraphenylenes, which due to their defined chemistry, have very low defect contents if compared to poly(phenylenevinylene)based materials. ${ }^{6}$

${ }^{a)}$ Electronic mail: f513ejwl@mbox.tu-graz.ac.at
The TSC depends on charge transport and, therefore, on many device parameters such as bulk carrier concentration and mobility, interface properties, etc. In contrast, photoluminescence (PL) -detected magnetic resonance (PLDMR) is a purely optical tool. It can probe any particular site in the material without requiring good charge transport or high collection efficiency. In conjugated polymers the charged defects are trapped polarons, the number of which we will denote as $N_{t}$. Their density and energetic position can be well characterized by TSC. On the other hand, with PLDMR one can characterize not only the density but more importantly the interaction of trapped polarons with the emissive SEs.

For our study, we chose two materials with comparable PL quantum yields of $30 \%$ in film but with very different structural properties: the amorphous soluble methylsubstituted ladder-type poly (para-phenylene) (PPP) ( $m$-LPPP) and the polycrystalline para-hexaphenyl (PHP). The synthesis of $m$-LPPP is described elsewhere, ${ }^{9}$ PHP is commercially available from Tokyo Chemical Industries and is used as delivered. $m$-LPPP samples were prepared for PLDMR measurements by dissolving the polymer powder in toluene in a quartz tube. The toluene was then evaporated leaving a $\sim 200$-nm-thick film on the inside walls of the tube. The sample was kept under dynamic vacuum of 1 $\times 10^{-6}$ mbar for $24 \mathrm{~h}$ before sealing the tube. PHP was evaporated on quartz substrates at a rate of $0.1 \AA / \mathrm{s}$ at a base pressure of $2 \times 10^{-6} \mathrm{mbar}$. To study the effects of photooxidation, the samples were irradiated by a $200 \mathrm{~W}$ xenon lamp for $\sim 30 \mathrm{~min}$ in air.

The sealed sample tubes were placed in the quartz dewar of an Oxford Instruments He gas-flow cryostat, enabling temperature control from 4 to $300 \mathrm{~K}$, inside an optically accessible $9.35 \mathrm{GHz} \mathrm{X}$-band microwave cavity. The PL was excited at 351 or $457.9 \mathrm{~nm}$ by a Pockels-cell-stabilized $\mathrm{Ar}^{+}$ laser. The PL and PLDMR spectra were measured using a $\mathrm{Si}$ 
a

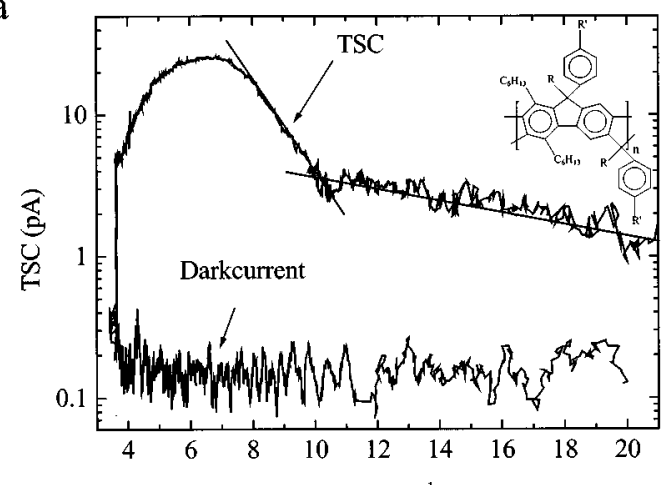

b

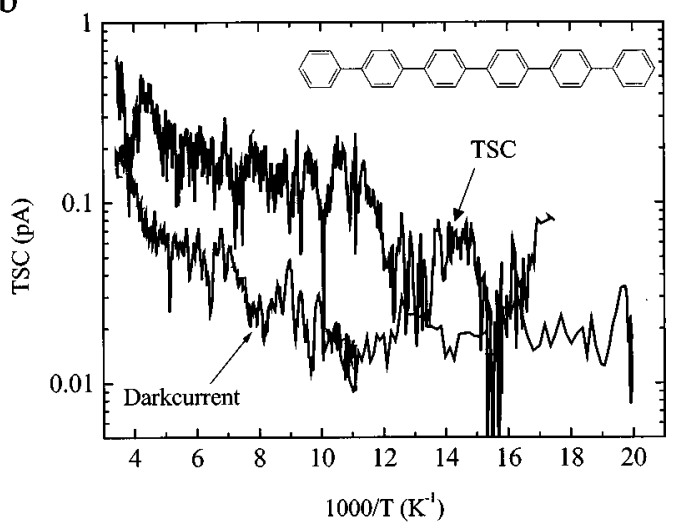

FIG. 1. Thermally stimulated and dark current of $m$-LPPP (a) and PHP (b). The inset in (a) shows the chemical structure of $m$-LPPP, $R=\mathrm{CH}_{3}, R^{\prime}$ $=\mathrm{C}_{10} \mathrm{H}_{21}, n \approx 25$; the inset in (b) shows the chemical structure of PHP.

photodiode, the laser line being blocked by a cutoff filter. The changes in the PL intensity, induced by the X-band microwaves at the applied dc magnetic field, were detected by feeding the photodiode output into a lock-in amplifier referenced to the microwave chopping frequency of $400 \mathrm{~Hz}$.

The $\sim 0.1 \mathrm{~cm}^{2}$ active area OLEDs used for TSC measurements were fabricated in a single-layer configuration using indium tin oxide and $\mathrm{Al}$ as the air stable electrodes. The polymer was dried for $24 \mathrm{~h}$ at $55^{\circ}$ and then stirred for $24 \mathrm{~h}$ in toluene to completely dissolve. The $\sim 150$-nm-thick active layers of the OLEDs were produced by spin coating $m$-LPPP from the $10 \mathrm{~g} / \mathrm{l}$ oxygen-free toluene solution and dried for 12 $\mathrm{h}$ in an argon box. PHP was evaporated with a rate of $0.1 \AA / \mathrm{s}$ at a base pressure of $10^{-6} \mathrm{mbar}$. Al was evaporated at a base pressure of $10^{-6}$ mbar after transferring the samples through air to the evaporator. All devices were stored under argon and only taken out for mounting in the cryostat.

TSC measurements were performed in the same cryostat used for PLDMR with a constant heating rate of $0.1-0.6 \mathrm{~K} / \mathrm{s}$. After filling all traps in the active layer of the OLED by $\sim 10$ min illumination with the xenon lamp at $50 \mathrm{~K}$, the TSC was driven by the $\sim 1 \mathrm{~V}$ built-in potential of the device and measured in a short-circuit mode using a Keithley electrometer. We note that no TSC could be detected for illumination times shorter than $3 \mathrm{~min}$.

Figures 1(a) and 1(b) show the TSC from $m$-LPPP and PHP devices, respectively, between 50 and $295 \mathrm{~K}$ with and without prior illumination (i.e., the latter being the dark current). As is clearly seen, in $m$-LPPP without prior illumination no significant TSC is detectable. In contrast, in PHP we still find a small TSC even without prior illumination. The rising conductivity with increasing temperature is due to the thermal generation of charge carriers due to unintentional doping of the PHP. The corresponding dark conductivity is of the order of $10^{-14} \mathrm{~S} / \mathrm{cm}$.

From the TSC curves we determine the lower limit of the trap density in the material by integration of the shortcircuit current over time for each TSC spectrum. Assuming that all traps are occupied at $50 \mathrm{~K}$ but only a fraction of the carriers are swept out, one obtains a lower limit for the number of traps $N_{t}$ from the relation:

$$
\int_{\text {total }} I d t \leqslant e N_{t},
$$

with $e$ the elementary charge. For all heating rates we determined a lower limit of the trap density of $2 \times 10^{16} \mathrm{~cm}^{-3}$ for $m$-LPPP and $1 \times 10^{14} \mathrm{~cm}^{-3}$ for PHP, which corresponds to $4 \times 10^{-5}$ traps per repeat unit in the polymer and $1 \times 10^{-7}$ traps per oligomer chain $\left(\rho=0.6 \mathrm{~g} \mathrm{~cm}^{-3}\right.$ for both materials).

The trapping energy can be estimated from the initial rise of the TSC with increasing temperature by

$$
\ln I=-\frac{E_{a}}{k_{B} T}+\text { const, }
$$

where $E_{a}$ is the activation energy. From the $m$-LPPP TSC spectrum we find two different activation energies: The behavior from 30 to $\sim 100 \mathrm{~K}$ yields $E_{a 1}=6 \mathrm{meV}$, and the rise below the main TSC peak yields $E_{a 2}=60 \mathrm{meV}$.

The number of contributing traps for each activation energy, calculated from Eq. (1), is given in Table I. For PHP such an analysis is difficult due to the absence of clear TSC features. However, from the rise of the TSC from 200 to 295 $\mathrm{K}$ one finds an approximate activation energy $E_{a}$ $\sim 120 \mathrm{meV}$.

Figure 2 shows the polaron PLDMR in pristine $m$-LPPP, photo-oxidized $m$-LPPP, and PHP films; the resonance amplitude $\Delta I / I$ in PHP is four times weaker than in $m$-LPPP. Photo-oxidization of $m$-LPPP increases $\Delta I / I$ by a factor of 3.5.

PLDMR probes the microwave-induced changes in the $\mathrm{PL}$ at the field for resonance. It is assumed that at resonance, one reduces the number of polarons acting as SE quenching

TABLE I. Trap depth $E_{a}$ and density $n_{t}$ in $m$-LPPP and PHP from TSC, $n_{\mathrm{tp}}$ density of trapped polarons from $\Delta I / I$ the PLDMR amplitude, for PHP ${ }^{\mathrm{a}}$ shows the trap density deduced from the dark current.

\begin{tabular}{lcccccc}
\hline \hline Sample & $\Delta I / I$ & $n_{\mathrm{tp}}\left(\mathrm{cm}^{-3}\right)$ & $E_{a 1}(\mathrm{meV})$ & $n_{t}\left(\mathrm{~cm}^{-3}\right)$ & $E_{a 2}(\mathrm{meV})$ & $n_{t}\left(\mathrm{~cm}^{-3}\right)$ \\
\hline$m$-LPPP & $3.3 \times 10^{-4}$ & $9 \times 10^{14}$ & 60 & $1.6 \times 10^{16}$ & 6 & $9 \times 10^{14}$ \\
Oxidized & $1.1 \times 10^{-3}$ & $\cdots$ & $\cdots$ & $\cdots$ & $\cdots$ & $\cdots$ \\
$m$-LPPP & & & & & & \\
PHP & $8 \times 10^{-5}$ & $\mathrm{a} 1.3 \times 10^{13}$ & 120 & $1.4 \times 10^{14}$ & $\ldots$ & $\ldots$ \\
\hline \hline
\end{tabular}




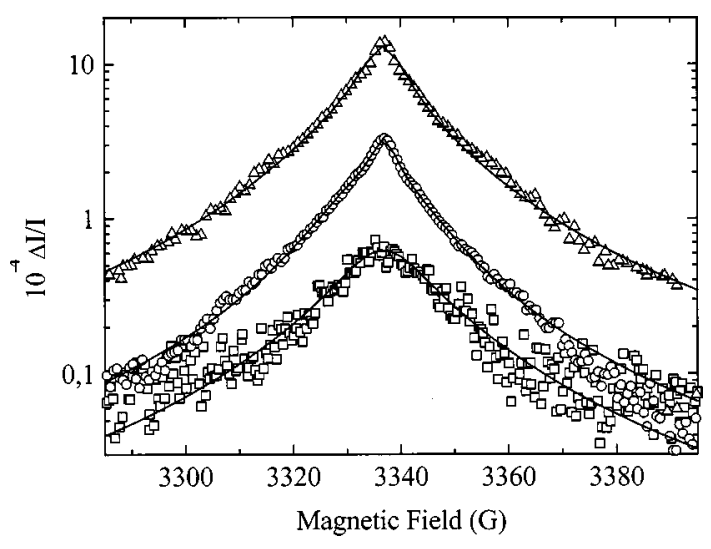

FIG. 2. Polaron PLDMR resonance $(\Delta I / I)$ of (circles) a pristine $m$-LPPP film, (triangles) a $m$-LPPP film after $30 \mathrm{~min}$. photo-oxidation, and (squares) a PHP film. The lines represent the double Lorentzian fits. $m$-LPPP, $\lambda_{\text {exc }}$ $=457.9 \mathrm{~nm}$, PHP $\lambda_{\text {exc }}=351 \mathrm{~nm}$, excitation density in both cases: 2 $\times 10^{23}$ photons $\mathrm{cm}^{-3} \mathrm{~s}^{-1}, T=20 \mathrm{~K}$, and microwave power $810 \mathrm{~mW}$.

centers, which consequently enhances the PL. ${ }^{10}$ As we show elsewhere, ${ }^{7}$ a proper choice of rate equations based on this mechanism leads to a model for the PL-enhancing polaron resonance, which is in quantitative agreement with the behavior of the PL and photoinduced absorption (PIA) in the same material. Simplified for the presented study, this model yields the relation

$$
\frac{\Delta I}{I}=\frac{\eta_{\mathrm{PL}} N_{\mathrm{SE}} \gamma \Delta_{\mathrm{MR}} N_{\mathrm{tp}}}{k_{r} N_{\mathrm{SE}}} \Rightarrow N_{\mathrm{tp}}=\frac{k_{r} \Delta I}{\Delta_{\mathrm{MR}} \eta_{\mathrm{PL}} \gamma I},
$$

where $N_{\mathrm{SE}}$ is the number of SEs, $k_{r}$ the radiative decay constant, $\eta_{\mathrm{PL}}$ the PL quantum yield, and $\gamma$ the annihilation parameter. The latter depends on the mobility of the SEs and is, therefore, a measure of the interaction between the two entities. $\Delta_{\mathrm{MR}} N_{\text {tp }}$ denotes the magnetic-resonance-induced, change in the polaron population $N_{\text {tp }}$. With Eq. (3) and $\Delta I / I$ from Fig. $2\left(\gamma=10^{-8} \mathrm{~cm}^{3} \mathrm{~s}^{-1}\right.$ for $m$-LPPP and PHP, $k_{r}$ $=10^{9} \mathrm{~s}^{-1}, \eta_{\mathrm{PL}}=0.3$ and $\Delta_{\mathrm{MR}}=2 \%$ for both systems ${ }^{7}$ ), one can calculate the number of trapped polarons to be 5 $\times 10^{15} \mathrm{~cm}^{-3}$ in $m$-LPPP and $10^{14} \mathrm{~cm}^{-3}$ in PHP for the used excitation density. Photo-oxidation decreased $\eta_{\mathrm{PL}}$ from $30 \%$ to $4 \%$ so that one finds a trap density of $10^{17} \mathrm{~cm}^{-3}$. The quantitative analysis shows that the PLDMR is influenced also by the diffusivity of SEs, whereas the TSC only depends on the concentration of trapped polarons.

PIA, TSC, ${ }^{11}$ and doping-induced absorption ${ }^{12}$ are suitable to detect and quantify charged defects, yielding the same activation energies. ${ }^{11}$ Comparing the PIA results obtained for different LPPPs demonstrated that chemical modification of LPPP alters the trap density by one order of magnitude, ${ }^{12}$ which is in accordance with a comparison of our present TSC results on low-defect-density $m$-LPPP with a high-defect-density LPPP (Ref. 11) (see Table I).

The very low trap density in PHP is also in full agree- ment with the fact that the polaron PIA bands of that material are weaker than the detection limit, ${ }^{13}$ which can also account for the relatively large mobility of $\sim 10^{-4} \mathrm{~cm}^{2} \mathrm{~V}^{-1} \mathrm{~s}^{-1}$ of carriers in PHP crystallites. ${ }^{14}$

However, utilizing the comparison of PLDMR and TSC, one can also probe the influence of charged defects on the PL yield. The number of trapped polarons determined from PLDMR and the number of trap states from TSC are much higher in both photo-oxidized and pristine $m$-LPPP than in PHP, and hence, in qualitative agreement with the fact that both signals are proportional to the number of trapped polarons $N_{\text {tp }}$. The fact that photo-oxidation increases the density of carbonyl groups, which increases the steady-state density of polarons, is well validated by the increase of $\Delta I / I$.

In conclusion, we have demonstrated that by comparing PLDMR and TSC measurements one can determine the density of traps to be $\geqslant 1.6 \times 10^{16}$ and $1.4 \times 10^{14} \mathrm{~cm}^{-3}$ in OLEDlike structures made of $m$-LPPP and PHP. For PHP we found that the interaction of the polarons with SEs was stronger than in $m$-LPPP, probably due to the higher diffusivity of the SEs, which leads to a relatively stronger nonradiative quenching of the latter by trapped polarons.

Financial support was provided by the FWF Project No. 12806, the BMWV, SFB Elektroaktive Stoffe, and ASPIRES at Virginia Tech. Ames Laboratory is operated by Iowa State University for the U.S. Department of Energy under Contract No. W-7405-Eng-82. This work was supported by the Director for Energy Research, Office of Basic Energy Sciences.

${ }^{1}$ G. Cerullo, S. Stagira, M. Nisoli, S. De Silvestri, G. Lanzani, G. Kranzelbinder, W. Graupner, and G. Leising, Phys. Rev. B 57, 12806 (1998), and references therein.

${ }^{2}$ S. Tasch, J. W. E. List, O. Ekström, W. Graupner, G. Leising, P. Schlichting, U. Rohr, Y. Geerts, U. Scherf, and K. Müllen, Appl. Phys. Lett. 71, 2883 (1997), and references therein.

${ }^{3}$ S. Stagira, M. Zavelani-Rossi, M. Nisoli, S. De Silvestri, G. Lanzani, C. Zenz, P. Mataloni, and G. Leising, Appl. Phys. Lett. 73, 2860 (1998).

${ }^{4}$ H. L. Gomes, P. Stallinga, H. Rost, A. B. Holmes, M. G. Harrison, and R. H. Friend, Appl. Phys. Lett. 74, 1144 (1999).

${ }^{5}$ S. M. Sze, Physics of Semiconductor Devices (Wiley, New York, 1981).

${ }^{6}$ H. Meyer, D. Haarer, H. Naarmann, and H. H. Hörhold, Phys. Rev. B 52, 2587 (1995).

${ }^{7}$ E. J. W. List, J. Partee, J. Shinar, W. Graupner, and G. Leising (unpublished).

${ }^{8}$ V. G. Kozlov, P. E. Burrows, G. Parthasarathy, and S. R. Forrest, Appl. Phys. Lett. 74, 1057 (1999).

${ }^{9}$ U. Scherf and K. Müllen, Makromol. Chem., Rapid Commun. 12, 489 (1991)

${ }^{10}$ Z. V. Vardeny and X. Wei, Mol. Cryst. Liq. Cryst. 256, 465 (1994); J. Shinar, Synth. Met. 78, 277 (1996).

${ }^{11}$ W. Graupner, G. Leditzky, G. Leising, and U. Scherf, Phys. Rev. B 54, 7610 (1996).

${ }^{12}$ W. Graupner, M. Mauri, J. Stampfl, G. Leising, U. Scherf, and K. Müllen, Solid State Commun. 91, 7 (1994).

${ }^{13}$ W. Graupner, F. Meghdadi, G. Leising, G. Lanzani, M. Nisoli, S. De Silvestri, W. Fischer, and F. Stelzer, Phys. Rev. B 56, 10128 (1997).

${ }^{14}$ A. Pogantsch, Diploma thesis, University of Technology Graz (1999). 